\title{
Chip Formation Mechanism Using Finite Element Simulation
}

\author{
Tahsin Tecelli Öpöz* - Xun Chen \\ Liverpool John Moores University, General Engineering Research Institute, UK
}

Prediction of chip form produced during machining process is an important work when considering workpiece surface creation and possible damage caused by chips generated during machining. The paper presents a set of new results of cutting chip formation from the latest finite element method (FEM) model development. Generally, three types of chips (continuous, serrated, and discontinuous chips) are generated during metal machining. The formation of these three types of chips is investigated in relation to various influential factors, such as rake angles and depth of cuts. Progressive damage model with a damage evolution criterion is employed into the FEM model to reduce mesh dependency. It has been demonstrated that finite element simulation is a good tool for the evaluation of chip formation in relation to operational parameters, tool settings as well as material properties.

Keywords: chip formation, finite element method, simulation, fracture energy, metal cutting

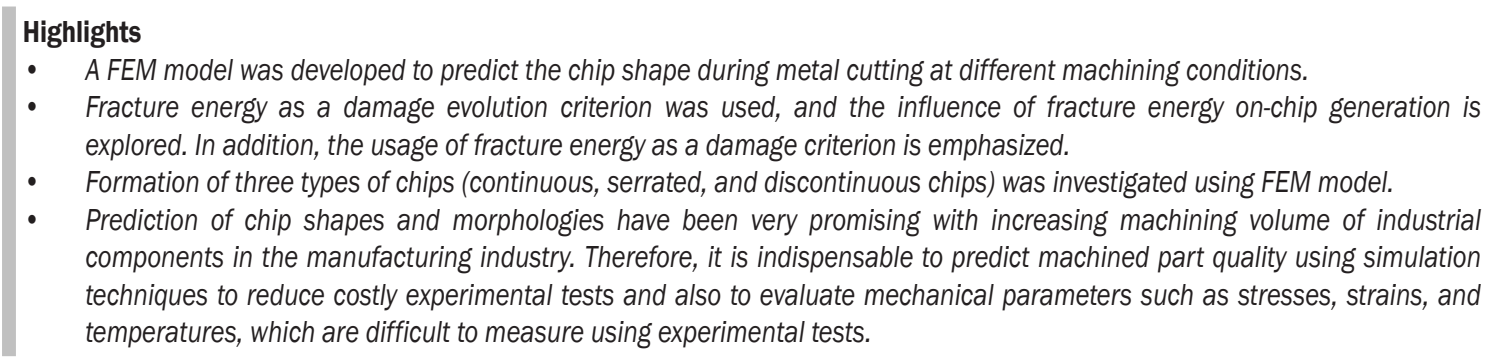

\section{INTRODUCTION}

Chip formation during machining processes is a material removal process in which the materials are removed from the workpiece in the form of numerous tiny chips. To understand chip formation regarding the chip types, shapes as well as the stress/strain distribution would help the prediction of cutting force and thermal behaviour to avoid unexpected vibration and thermal damage. To do this, the finite element models of machining processes are very popular for simulating chip formation [1] to [15]. The reason is to minimize labour cost and save time by reducing expensive experimental tests. In contrast, the estimation of some physical phenomena such as the distribution of stress and strain under machining conditions is quite difficult to accomplish with experimental tests. Finite element method (FEM) provides a convenient method to visualise material performance under different machining conditions. Therefore, the prediction of chip types and chip shapes under different operating conditions is one of the significant benefits provided by FEM simulations.

In metal cutting processes, often three types of chip formation occur (continuous chips, serrated chips, and discontinuous chips), which are produced as a result of the cutting deformation mechanism, operating parameters, and workpiece mechanical and thermal properties. The continuous chip is often considered to be an ideal chip that generates stable cutting forces; however, it is not desired for automated machining because the continuous chips may obstruct the machining process, which may lead to unpredictable damage on the machined surface, cutting tool or machine tool. To minimize these problems, serrated chips that are easier to break and remove are preferred during machining [1] and [2], so, predicting the cutting conditions which lead to a serrated chip has become increasingly important.

In some literature, serrated chips are called sawtooth shape or continuous segmented chips. Increased segmentation on continuous chips eventually leads to serrated chips. It is common knowledge that segmentation during chip formation is triggered by two phenomena: the formation of the adiabatic shear band and the crack initiation mechanism in a primary shear zone. Adiabatic shear banding is known as the localization of the plastic deformation into the narrow bands which occur during the high-rate plastic deformation and often lead to shear fracture [3]. Serrated chips are formed as a result of adiabatic shear bands [4]. In addition to adiabatic shear band deduced segmentation, the serrated chip is formed when a chip fractures at the primary deformation zone due to overstrain and the interfaces of the chip segments are welded immediately after the fracture by compression 
and high chemical activity, and a crack is generated according to material failure criterion. After the crack initiation, it is propagated in the direction of the shear zone into the chip, which enables segmentation [5]. The generation of segmentation in FEM modelling is achieved by employing either failure criterion or modified constitutive model regarding strain, strain rate, and temperature as a function of flow stress into the finite element model. Deployment of flow stress without failure criterion is highly necessary, coupled with continuous adaptive remeshing to mitigate the distortion of the element taking place due to high plastic deformation at the primary and secondary deformation zones, ultimately, adaptive remeshing also enable to complete solution without termination of the model caused by excessive element distortion during computation.

Aurich and Bil [5] developed a 3D chip formation model by introducing Rhim's flow stress equations to account for thermal softening, adaptive remeshing to deal with element distortion and fracture mechanic-based chip separation criterion. They found a significant effect of the applied method on predicted chip shape; adaptive remeshing alone leads to continuous chip, thermal softening generates brief segmentation, and fracture leads to severe segmentation. Calamaz et al. [6] studied the influence of the strain softening phenomenon and friction law on the shear location generating serrated chips. Bäker et al. [7] developed a two-dimensional orthogonal cutting process considering adiabatic shearing effects and generated segmented chips by employing a remeshing technique to the model.

Segmentation of chips is also dependent on operating parameters; segmented continuous chips are often produced at high cutting speeds [8] and the degree of segmentation increases with increasing feed or uncut chip thickness [4]. The tendency to form segmented chips is found to be smaller at the lower cutting speed [7]. This indicates the complexity of chip formation with a large number of influential factors. Regarding material property effects, the lower the elastic modulus is, the higher the degree of segmentation and segmentation time would be. A possible explanation is that a larger amount of stored elastic energy eases the shearing, or that plastic shearing is preferred as elastic deformation of the region left of the shear band is energetically unfavourable.

The earliest FEM simulation on chip formation was conducted by Strenkowski and Carroll [9], and they achieved serrated chips by using the chip separation criterion. To date, chip separation was achieved either by previously defined separation criteria or by the fully plastic flow of material using an adaptive remeshing technique employed to the FEM model. Finite element analysis of chip formation can be modelled either by using Eulerian formulation or Lagrangian formulation. In a Eulerian-based model, no need to define chip separation criterion exists; cutting is simulated from the steady state, but it is required to define the initial chip shape, so it is not very realistic for investigating machining chip formation. Conversely, the Lagrangian formulation can allow simulating chip formation without defining initial chip shape from incipient to the steady state. It gives more realistic results as a prediction of chip geometry and other machining-deduced phenomenal parameters, such as stress, strain, and force. However, the Lagrangian formulation needs a chip separation criterion to enable chip separation from the workpiece [10]. Huang and Black [11] evaluated available chip separation criteria and divided them into two categories: (a) physical criteria such as effective plastic strain and strain energy density; and (b) geometrical criteria such as distance tolerance. They concluded that neither the geometrical nor physical criteria simulated machining process correctly and suggested a combination of geometric and physical criteria for FEM simulation of machining. Often, parting lines, or sacrificial layer, are used together with a failure criterion to allow chip separation from the workpiece when the updated Lagrangian formulation is used.

In early FEM models of chip formation, a node release technique was based on distance tolerance; effective plastic strain and strain energy density [10] and [12] were employed to the model. According to node release technique, an element in front of the tool is separated following the nodal release when the defined criterion is satisfied. Recently, three other methods have been utilized to generate chips: material failure (damage) models based on fracture mechanics, flow stress models which take thermal softening and straining hardening into account with continuous adaptive remeshing, and ArbitraryLagrangian-Eulerian (ALE) adaptive meshing. The first one considers the fracture mechanic concept to initiate a crack and followed by crack growth to form chips according to degradation criterion. The last two produce chips due to the plastic flow of material over the tool tip without crack formation. Moreover, the material failure model and adaptive remeshing are used together to achieve further improvement in chip formation in some models. Particularly, for the formation of serrated chips, the inclusion of a material 
failure model and adaptive remeshing method into FEM model is highly crucial.

Shet and Deng [13] developed the orthogonal cutting process under plane strain conditions in Abaqus/Explicit. Explicit FEA is generally preferred in cutting simulation because explicit analyses guarantee to converge. Large deformation was prevented by an updated Lagrangian formulation. Movahhedy et al. [14] developed an orthogonal cutting model by using ALE approach with strain rate and temperature dependent constitutive equations. Özel and Zeren [15] developed a finite element model with ALE adaptive meshing without using chip separation criteria; chip was generated by the plastic flow of workpiece material. Sima and Özel [4] simulated the serrated chips without using damage or failure models by adiabatic shearing due to temperature -dependent flow softening with continuous remeshing in Deform 2D.

A progressive failure model needs a properly defined damage evolution technique. In Abaqus, damage evolution can be conducted by defining either equivalent plastic displacement or fracture energy dissipation [16] to [19]. Ambati and Yuan [17] investigated the mesh-dependence in cutting simulation by using the progressive failure model with plastic displacement as the damage evolution criterion. They analysed various chip shapes by using different plastic displacement values and found that chip transition occurs from continuous to segmentation with increasing feeds. Mabrouki et al. [2] investigated the characteristics of chip morphology and chip microstructures during cutting operation under high loading. Mabrouki et al. [18] also investigated the dry cutting of an aeronautic aluminium alloy and developed the numerical model based on the Johnson-Cook law incorporating with material damage evolution by using a fracture energy model. The feed rate and cutting velocity were used as the simulation variables. Their experimental results showed that the higher cutting speed results in increasing segmentation frequency. Chen at al. [20] employed energy density based failure criteria to investigate the flow stress and failure strain behaviour in orthogonal cutting of aluminium alloys.

In metal cutting, simulations results are often considered as dependent on mesh size so called mesh sensitivity. In cutting simulation at large deformation when using a progressive failure model in Abaqus, element characteristics, length could be increased to reduce the mesh dependency [21]. In the determination of element size, there are two main constraints that should be taken into account: (1) mesh element size should be relatively high to obtain a reasonable computation time and (2) the results should be similar to the experimental results in terms of chip morphology and cutting force generation [21]. It must be noted that the influence of mesh size (mesh sensitivity) is not investigated in this paper; a constant element mesh size of $1 \mu \mathrm{m}$ in length is used for the chip formation.

\section{FINITE ELEMENT SIMULATION}

\subsection{Simulation Environment and Boundary Conditions}

In this paper, all simulations are performed in Abaqus/ Explicit FEM software. CPE4RT elements, 4-node bilinear displacement, and temperature, reduced integration with hourglass control, are used in the workpiece model. Element size in cutting region (top section of the workpiece) is $1 \mu \mathrm{m}$ in length. Although the cutting tool is meshed, a rigid body constrained is applied to the cutting tool in the simulations. Cutting tool speed of $300 \mathrm{~m} / \mathrm{min}$ is used for all simulations. Workpiece boundary conditions are shown in Fig. 1.

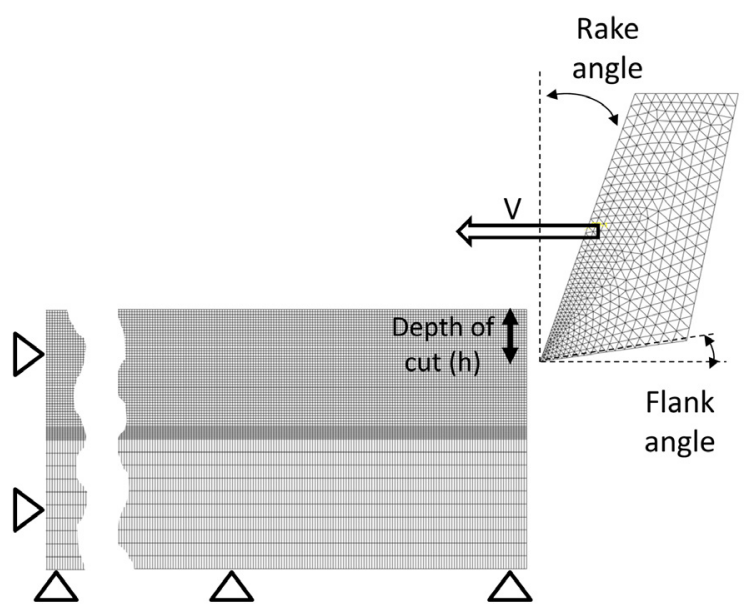

Fig. 1. An example of tool and workpiece model with positive tool rake angle

The penalty contact method with a constant friction coefficient $(\mu=0.2)$ is applied between the tool and workpiece. Johnson-Cook (JC) flow stress parameters are given in Table 1. The simulation is modelled considering the adiabatic heating effect on the analysis. Element deletion technique is used to allow element separation to form a chip. In the present study, no sacrificial layer is used to allow chip separation. The ALE adaptive meshing technique is used to maintain the mesh quality throughout the simulation and to reduce element distortion in cases of 
extreme deformation. Damage criterion and element deletion is applied to entire workpiece elements, so that it might be possible to simulate crack initiation along a chip's primary shear region. However, one drawback without a sacrificial layer, the simulation might be terminated if the elements distortions increase excessively due to inconvenient damage parameters. In addition, without a sacrificial layer, some elements in the chip separation region where the tool comes into contact with workpiece extend too much as seen black lines in Fig. $5 \mathrm{c}$ in a later section.

Table 1. JC flow stress parameters for workpiece (A2024-T351) [18]

\begin{tabular}{lc}
\hline Parameters & values \\
\hline$A[\mathrm{MPa}]$ & 352 \\
\hline$B[\mathrm{MPa}]$ & 440 \\
\hline$n$ & 0.42 \\
\hline$C$ & 0.0083 \\
\hline$m$ & 1 \\
\hline Density, $\rho\left[\mathrm{kg} / \mathrm{m}^{3}\right]$ & 2700 \\
\hline Elastic modulus, $E[\mathrm{GPa}]$ & 73 \\
\hline Poisson's ratio & 0.33 \\
\hline Specific heat, $C_{p}\left[\mathrm{~J} /\left(\mathrm{kg} \cdot{ }^{\circ} \mathrm{C}\right)\right]$ & $C_{p}=0.557 \mathrm{~T}+877.6$ \\
\hline Expansion, $\alpha_{d}\left[\mu \mathrm{m} /\left(\mathrm{m} \cdot{ }^{\circ} \mathrm{C}\right)\right]$ & $\alpha_{d}=8.9 \times 10^{-3} \mathrm{~T}+22.2$ \\
\hline$T_{\text {melt }}\left[{ }^{\circ} \mathrm{C}\right]$ & 520 \\
\hline$T_{\text {room }}\left[{ }^{\circ} \mathrm{C}\right]$ & 25 \\
\hline Heat fraction coefficient & 0.9 \\
\hline
\end{tabular}

\subsection{Constitutive Model}

In the present study, a rate-dependent JC model [22] was used. Rate dependent yield is required to model materials' yield behaviour accurately when the yield strength depends on the strain rate. The JC hardening method describes the flow stress of a material with the product of strain, strain rate, and temperature effect is defined in Eq. (1).

$\bar{\sigma}=\left[A+B(\bar{\varepsilon})^{n}\right]\left[1+C \ln \left(\frac{\dot{\bar{\varepsilon}}}{\dot{\overline{\varepsilon_{0}}}}\right)\right]\left[1-\left(\frac{T-T_{\text {room }}}{T_{\text {melt }}-T_{\text {room }}}\right)^{m}\right]$,

where $\bar{\sigma}$ is the equivalent stress, $A$ is the initial yield strength $[\mathrm{MPa}]$ of the material at room temperature, $B$ is the hardening modulus [MPa], $\bar{\varepsilon}$ is the equivalent plastic strain, $n$ is the work-hardening exponent, $C$ is the coefficient dependent on the strain rate, $\dot{\bar{\varepsilon}}$ is the equivalent plastic strain rate, $\dot{\bar{\varepsilon}}_{0}$ is the reference strain rate, $m$ is the thermal softening coefficient. $T_{\text {room }}$ and $T_{\text {melt }}$ represent the room temperature and melting temperature, respectively. The flow stress curves of
Johnson-Cook model for the material properties used in the simulation are shown in Fig. 2.

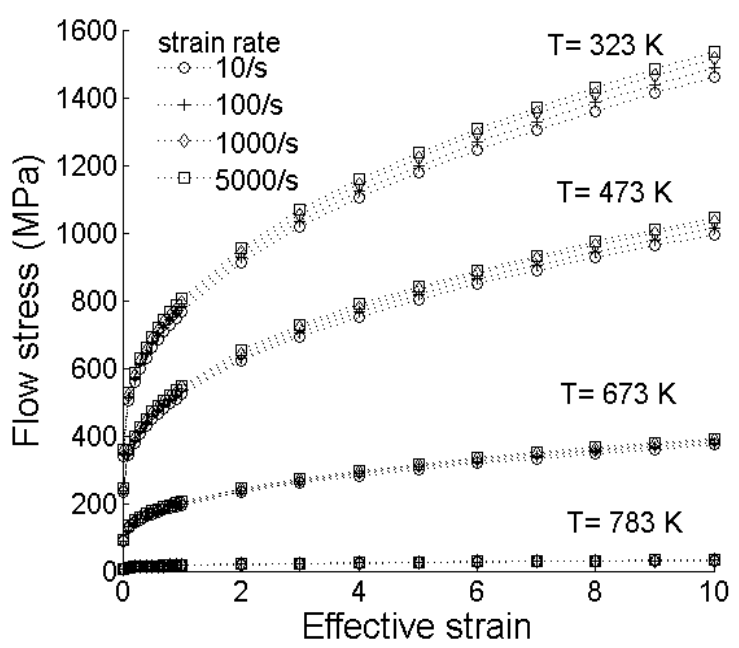

Fig. 2. Flow stress curves with temperature and strain rate data using JC model

\subsection{Damage Initiation}

The damage model proposed by Johnson and Cook [23] is used in conjunction with JC flow stress model. The JC damage model is suitable for high strain rate deformation, such as high-speed machining. The JC damage model was claimed to result in more realistic simulations compared to other models (e.g. Wilkins, the maximum shear stress, the modified Cockcroft-Latham, the constant fracture strain, and the Bao-Wierzbicki fracture models) [24]. According to JC damage criterion, the general expression for the fracture strain is given in Eq. (2).

$$
\begin{aligned}
\bar{\varepsilon}^{f} & =\left(D_{1}+D_{2} \exp D_{3} \frac{\sigma_{m}}{\bar{\sigma}}\right)\left(1+D_{4} \ln \frac{\dot{\bar{\varepsilon}}}{\dot{\bar{\varepsilon}}}\right) \times \\
& \times\left[1+D_{5}\left(\frac{T-T_{\text {room }}}{T_{\text {melt }}-T_{\text {room }}}\right)\right],
\end{aligned}
$$

JC damage parameters represent: $D_{1}$ initial failure strain, $D_{2}$ exponential factor, $D_{3}$ triaxiality factor, $D_{4}$ strain rate factor, and $D_{5}$ temperature factor, where $\bar{\varepsilon}^{f}$ the equivalent strain to fracture is, $\sigma_{m}$ is the average of the three normal stresses and $\bar{\sigma}$ is the von Mises equivalent stress.

Damage initiation begins according to the standard damage law,

$$
w=\sum \frac{\Delta \bar{\varepsilon}}{\bar{\varepsilon}^{f}},
$$


where $\Delta \bar{\varepsilon}$ is the accumulated increment of equivalent plastic strain during an integration step, $w$ is damage parameter to initiate failure when it equals to 1 . Due to the use of progressive damage, damage initiation is followed by damage evolution criterion which governs the propagation of damage until an ultimate failure happens.

The JC damage parameters used for the present study are given in Table 2. The temperature factor $D_{5}$ is null which means that the temperature has no effect on the damage initiation during the cutting of the aluminium alloy A2024-T351 [18].

Table 2. JC damage parameters for A2024-T351 [18]

\begin{tabular}{ccccc}
\hline$D_{1}$ & $D_{2}$ & $D_{3}$ & $D_{4}$ & $D_{5}$ \\
\hline 0.13 & 0.13 & -1.5 & 0.011 & 0 \\
\hline
\end{tabular}

\subsection{Damage Evolution Based on Fracture Energy Dissipation}

The stress-strain relationship defined in the material properties table cannot represent the material behaviour properly after the initiation of damage. Usage of stress-strain relation through damage propagation results in significant mesh dependency due to strain localization [25]. To mitigate the mesh dependency, a damage evolution technique with linear softening based on the fracture energy model proposed by Hillerborg et al. [26] is used in this paper. Hillerborg defines fracture energy, $G_{f}$ (with a unit of $\mathrm{J} / \mathrm{m}^{2}$ or $\mathrm{N} / \mathrm{m}$ in Abaqus), which is required to obtain a unit area of crack as a material parameter. This governs the softening of material according to a stressdisplacement response after damage initiation rather than a stress-strain response [25]. To determine the stress-displacement response, a characteristic length $(L)$, which is the typical length of line in the firstorder element associated with an integration point, is defined. Hillerborg's fracture energy is defined in Eq. (4).

$$
G_{f}=\int_{\bar{\varepsilon}_{0}^{p l}}^{\bar{\varepsilon}_{f}^{p l}} L \sigma_{y} d \bar{\varepsilon}^{p l}=\int_{0}^{u_{f}^{p l}} \sigma_{y} d \bar{u}^{p l},
$$

where, $\bar{u}^{p l}$ is the equivalent plastic displacement as the fracture work conjugate of the yield stress after the onset of damage. Linear evolution of the damage is assumed. Before damage initiation $\bar{u}^{p l}=0$; after damage initiation $\bar{u}^{p l}=L \bar{\varepsilon}^{p l}$. In the Abaqus FEM model, fracture energy is an input parameter which is required to be known before simulation. Thus, following the damage initiation, the damage variable increases according to Eq. (5).

$$
D=\frac{L \bar{\varepsilon}^{p l}}{\bar{u}_{f}^{p l}}=\frac{\bar{u}^{p l}}{\bar{u}_{f}^{p l}}
$$

where the equivalent plastic displacement at failure is computed as in Eq. (6).

$$
\bar{u}_{f}^{p l}=\frac{2 G_{f}}{\sigma_{y 0}},
$$

where $\sigma_{y 0}$ is the value of yield stress at the time when the failure criterion is reached. Stress-strain behaviour of a material undergoing damage is illustrated in Fig. 3. The solid curve in the figure represents the damaged stress-strain response, while the dashed curve is the response in the absence of damage. In the figure, $\sigma_{y 0}$ and $\bar{\varepsilon}_{0}^{p l}$ are the yield stress and equivalent plastic strain at the onset of damage, and $\bar{\varepsilon}_{f}^{p l}$ is the equivalent plastic strain at failure; that is when the overall damage variable reaches the value $D=1$. Then the concerned element is removed from the computation when $D=1$ in every integration point, with the element deletion technique provided by Abaqus software. Overall damage variable, $D$, can be obtained from the simulation output as a degradation variable, $S D E G$, and can be set to a value lower than 1 considering the course of simulation [20]. The SDEG value in this study is a default 0.99 in Abaqus to ease the element deletion.

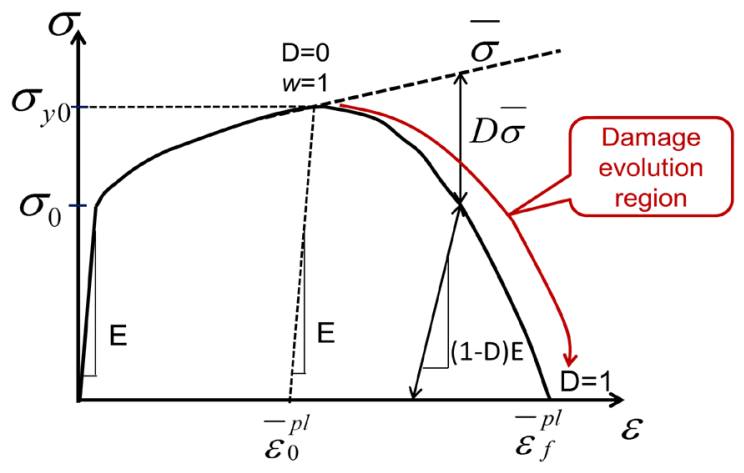

Fig. 3. Stress-strain curve illustrates damage evolution for progressive damage model [20]

The strength of the material along the curve can be calculated by Eq. (7).

$$
\sigma=(1-D) \bar{\sigma}
$$

where $\sigma$ and $\bar{\sigma}$ are the effective and apparent stress and $D$ is the accumulated damage. In addition, the elastic modulus decreases due to damage [26]. Elastic modulus after damage initiation $\tilde{E}$ can be calculated by Eq. (8). 


$$
\tilde{E}=(1-D) E \text {. }
$$

The difficulty in damage evolution is due to the requirement of a previously known fracture energy value as an input parameter. The fracture energy required for the damage evolution can be determined by Eq. (9).

$$
G_{f}=K_{I C}^{2}\left(\frac{1-v^{2}}{E}\right) \text { (Plane strain). }
$$

Evaluated fracture energy is related to fracture toughness $K_{I C}$, Young's modulus $E$, and Poisson's ratio $v$. As an important criterion, fracture toughness $\left(K_{I C}\right)$ indicates the resistance to crack growth and can be used to predict fracture initiation. High plastic deformation in the machining process causes variation in fracture toughness. He and $\mathrm{Li}$ [27] investigated the influences of fracture toughness on strengthening and degrading aspects. With the increasing number of defects (micro-cracks and growth), some physical characteristic of materials are also changed such as elastic modulus as recalculated in Eq. (8) and toughness as well.

Measurements of $K_{I C}$ are made using specimens containing very sharp fatigue pre-cracks. However, there are constraints on specimen dimension and crack length. These limitations make it difficult to obtain valid $K_{I C}$ values for materials [28]. The experimental evidence shows that the fracture toughness of metals is dependent on the size of the specimen during the test. Although at the bigger size of the test specimen, fracture toughness was calculated as being more stable, whereas, it is dramatically decreasing while the test specimen size is decreasing to lower values as shown in Fig. 4. When chip formation is considered, the chip size is relatively small where the fracture initiated and propagated. So it is not realistic to use $K_{I C}$ values measured at nominal test specimen size, which is relatively higher than required for chip formation. In addition, $K_{I C}$ is not a constant property; it is varying depending on the fracture mode of material including the micro-crack formation, element dislocation, and subsequent crack growth along the fracture line. Mabrouki et al. [18] the employed fracture energy method into the FEM model by using $K_{I C}$ values measured at a nominal size to estimate the fracture energy required as a damage evolution criterion. $G_{f}$ values defined in [18] is $16711 \mathrm{~N} / \mathrm{m}$ for the opening mode of fracture and $8251 \mathrm{~N} / \mathrm{m}$ for the shearing mode of fracture, which are estimated from Eq. (9). A detail explanation of opening and shearing mode is provided in [18]. In this paper, fracture energy required for damage evolution is not determined from $K_{I C}$ values; a range of $G_{f}$ values from $250 \mathrm{~N} / \mathrm{m}$ to $20000 \mathrm{~N} / \mathrm{m}$ is used to emphasize the influences of $G_{f}$ value on the chip type and morphology. Therefore, significant changes have been observed in the chip type and shape.

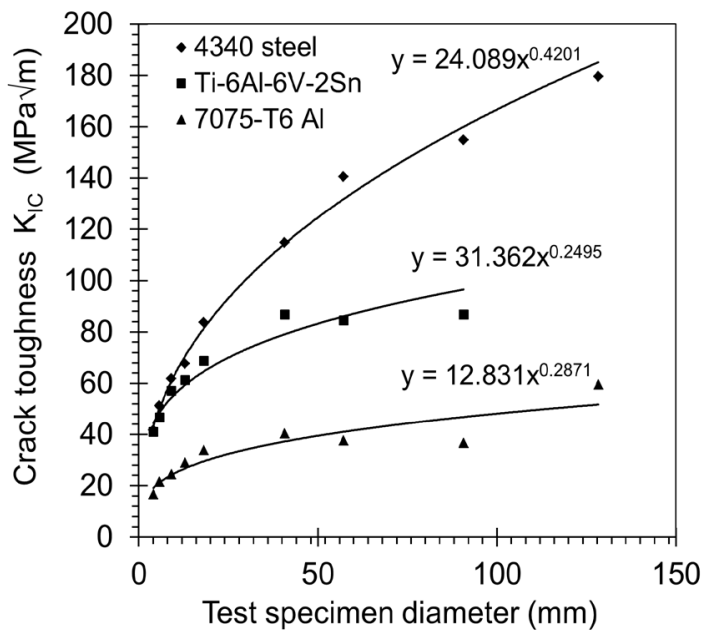

Fig. 4. Crack toughness $\left(K_{I C}\right)$ versus section size [29]

\subsection{Temperature Rise Due to Adiabatic Process}

In high-speed machining, heat produced due to local energy dissipation may not have adequate time to diffuse away, and local heating will take place in the active plastic zones and sliding frictional interface. Thus, the temperature rise in the chip can be estimated with the adiabatic heating condition. The temperature increase is calculated directly at the material integration points according to the adiabatic thermal energy increases caused by inelastic deformation [13], [16] and [30]. Heat conduction has not a contribution in an adiabatic analysis. Volumetric heat flux due to plastic straining can be calculated by Eq. (10).

$$
\dot{q}=\eta \dot{\sigma} \varepsilon,
$$

where $\dot{q}_{p}$ is the heat flux that is added to the thermal energy balance, $\eta$ is the inelastic heat fraction (assumed constant as a default value of 0.9 in Abaqus), $\sigma$ is the effective stress, and $\dot{\varepsilon}^{p}$ is the plastic strain rate. Also, the heat equation solved at each integration point is given in Eq. (11).

$$
\rho C_{p} \frac{\Delta T_{p}}{\Delta t}=\dot{q}_{p},
$$

where $\rho$ is the material density and $C_{p}$ is the specific heat. Considering both equations shown above, the 
local temperature rise due to plastic deformation can be given in Eq. (12).

$$
\Delta T_{p}=\eta \frac{\sigma \dot{\varepsilon}^{p} \Delta t}{\rho C_{p}}
$$

Similarly, heat generated by friction forces (Eq. (13)) lead to a rise in temperature $\Delta T_{f}$ during a period of $\Delta t$.

$$
\dot{q}_{f}=\eta_{f} J \tau \dot{\gamma}=\rho C_{p} \frac{\Delta T_{f}}{\Delta t} .
$$

Then temperature rise due to sliding friction between tool and chip interfaces is determined by Eq. (14):

$$
\Delta T_{f}=\eta_{f} \frac{J \tau \dot{\gamma} \Delta t}{\rho C_{p}},
$$

where, $\eta_{f}$ is the fraction of dissipated energy caused by friction and assumed as $0.9, J$ the equivalent heat conversion factor, $\tau$ shear stress computed by Coulomb's law and $\dot{\gamma}$ slip strain rate.

\section{FEM SIMULATION RESULTS}

Chip formation simulations were performed with gradually increasing fracture energy, $G_{f}$, two different depth of cuts $\left(h_{1}=20 \mu \mathrm{m}\right.$ and $\left.h_{2}=50 \mu \mathrm{m}\right)$ and four rake angles $\left(\gamma_{1}=22^{\circ}, \gamma_{2}=0^{\circ}, \gamma_{3}=-30^{\circ}, \gamma_{3}=-45^{\circ}\right)$ in Abaqus/Explicit.

\subsection{Fracture Energy Influence on Chip Formation}

A simulation with gradually increasing fracture energy, $G_{f}$, was performed to demonstrate the influence of fracture energy which is used as damage evolution criterion. Due to difficulties in knowing the exact value of the fracture energy at the smaller thickness, chip formation have been simulated with different values of fracture energy $(250 \mathrm{~N} / \mathrm{m}, 2500$ $\mathrm{N} / \mathrm{m}, 10000 \mathrm{~N} / \mathrm{m}$, and $20000 \mathrm{~N} / \mathrm{m}$ ) while uncut chip thickness remains $20 \mu \mathrm{m}$. The results shown in Fig. 5 demonstrate that increasing fracture energy $(2500 \mathrm{~N} / \mathrm{m}, 10000 \mathrm{~N} / \mathrm{m}$ and $20000 \mathrm{~N} / \mathrm{m}$ ) leads to the generation of the continuous chips, as shown in Figs. $5 b-d$ while lower values of fracture energy $(250 \mathrm{~N} / \mathrm{m})$ results in discontinuous chip generation as shown in Fig. 5a. Furthermore, chip shapes tend to be straighter with increasing fracture energy (Figs. $5 \mathrm{~b}$ tod) while lower fracture energy result in more curled chips, as shown in Fig. 5b. Therefore, fracture energy is one of the key factors as a damage evolution criterion to determine the chip generation behaviour during cutting simulation.
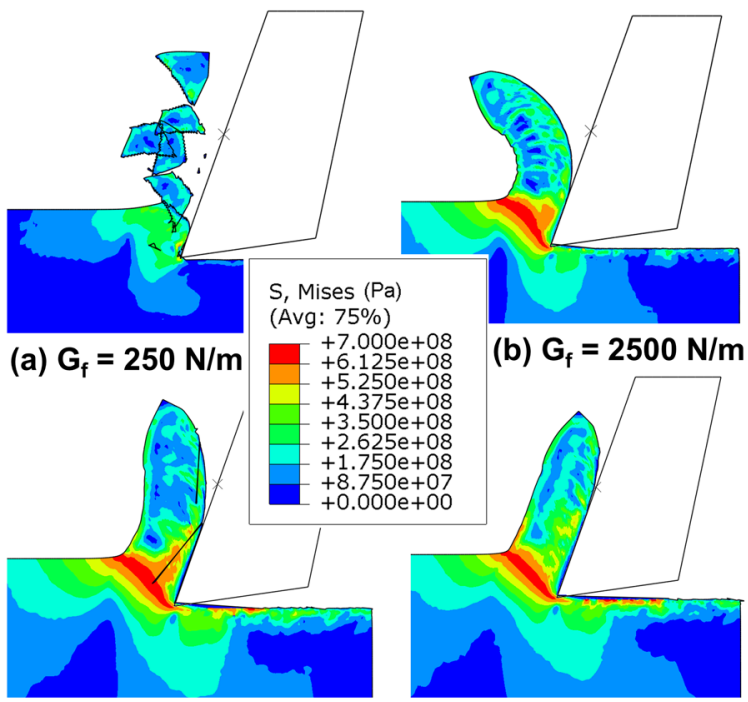

(c) $G_{f}=10000 \mathrm{~N} / \mathrm{m}$

(d) $G_{f}=20000 \mathrm{~N} / \mathrm{m}$

Fig. 5. Chip simulations with varying fracture energy criterion for damage evolution $(h=20 \mu \mathrm{m})$

\subsection{Influence of Depth of Cut on Chip Type}

The depth of cut is also one of the influential parameters for the determination of chip type. To show the depth of cut influence, two different simulations with different depths of cut were made. A continuous chip without forming segmentation along the chip free edge is generated when uncut chip thickness is set to $20 \mu \mathrm{m}$ as shown in Fig. 6a. In contrast, the serrated chip is generated when the depth of cut is about 50 $\mu \mathrm{m}$ under the same simulation conditions as shown in Fig. 6b. The reason of serrated chip formation at higher uncut chip thickness is probably shear stress developed in the primary shear zone increasing dramatically due to intensive straining in this region. With the increasing strain at the primary shear zone, material strength is weakened due to the predefined

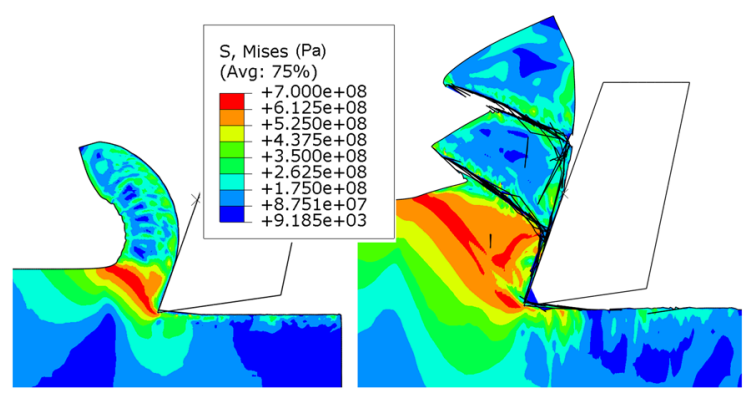

(a) $\mathrm{h}=\mathbf{2 0} \mu \mathrm{m}$

(b) $\mathbf{h}=\mathbf{5 0} \boldsymbol{\mu m}$

Fig. 6. Chip formation with different depth of cuts

$$
\left(G_{f}=2500 \mathrm{~N} / \mathrm{m}\right)
$$


failure criterion and crack initiation in the primary shear region. As a result, each segment formed due to plastic flow and formed sharp-edged serrated chips as shown in Fig. 6b. Experimental results obtained in [31] support the present simulations, i.e. the tendency to produce segmented chips is increasing with increased feed.

\subsection{Influence of Rake Angle on Chip Formation}

The formation of continuous, serrated and discontinuous chips was simulated by using the different tool rake angles. Though previous research [4] did not show a significant effect of rake angle on the degree of segmentation, it has a significant influence in low values of the depth of cut. Fig. 7 shows that the rake angle plays a crucial role in determining chip type. A continuous chip with a little segmentation is generated with a positive rake angle of $22^{\circ}$ (Fig. $7 \mathrm{a})$; a serrated or saw-tooth chip is generated with a zero rake angle tool (Fig. 7b); a discontinuous chip is generated with a negative rake angle of $-30^{\circ}$ (Fig. $7 \mathrm{c}$ ); and a different discontinuous chip with two split parts is generated with a negative rake angle of $-45^{\circ}$ (Fig. 7d). Obviously, the tendency from continuous to discontinuous chip formation is increasing while the rake angle is moving from positive to negative values. As a result of the negatively increasing tool rake face, compression on the chip increases. This higher compression results in tearing stress increasing in the primary shear region, which in turn promotes segmentation, then eventually separation of each

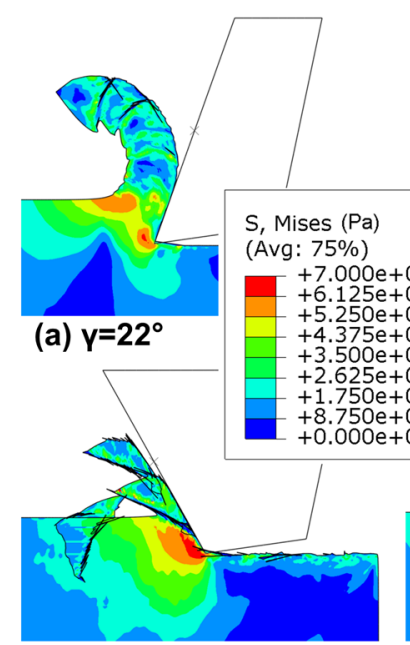

(c) $\mathrm{Y}=-30^{\circ}$

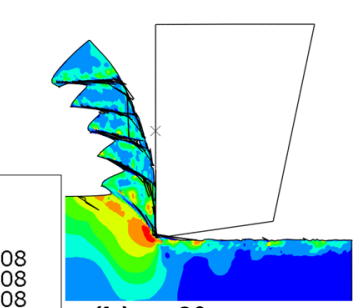

(b) $\mathrm{Y}=0^{\circ}$

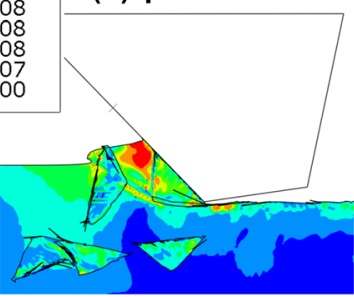

(d) $\mathrm{y}=-45^{\circ}$
Fig. 7. Chip shape alteration with varying rake angles $\left(G_{f}=1500 \mathrm{~N} / \mathrm{m}\right.$ and $\left.h=20 \mu \mathrm{m}\right)$ segment. Since material strength in the primary shear region decreased with increasing straining, ultimate fracture occurs when the predefined damage evolution criterion is satisfied. Experimental results in [31] confirm that the chip shape changes from continuous to segmented chip and also segmentation degree increases with the smaller rake angle. In [31], there was not any experimental result for the negative rake angle.

\subsection{Chip Fracture Development due to Increasing Chip Length}

Chip fracture is observed in serrated chip formation simulation with simulated chip length is increasing with increasing simulation time as shown in Fig. 8. After reaching a certain length, the chip is fractured from the weak residual shear zone since with the increasing chip length chip curvature increases than elements in the weakened residual shear zone no longer withstand carrying the frontier portion of the chip then fracture will occur as shown in Fig. 8b.

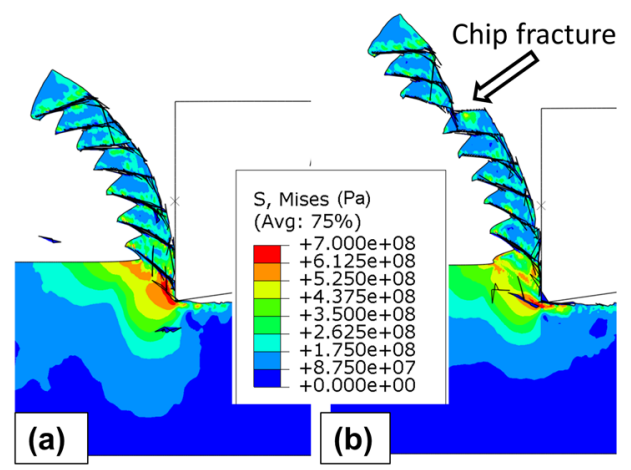

Fig. 8. Chip fracture during serrated chip formation at a) $2.85 \mathrm{e}-5 \mathrm{~s}$ and b) $3.42 \mathrm{e}-5 \mathrm{~s},\left(G_{f}=1500 \mathrm{~N} / \mathrm{m}, h=20 \mu \mathrm{m}, \gamma=0^{\circ}\right)$

\subsection{Temperature Changes in Chip Formation}

Temperature increases across the shear band and high deformation area due to energy consumption in plastic deformation and friction. It is observed that temperature rises mainly in the chip region, with maximum values localized along the tool-chip interface and at the fracture zone. Fig. 9 demonstrates temperature variation on chips with three different tool rake angles. Temperature-affected region between the workpiece top surface layer and the sub-surface layer increases while the tool rake face angle changes from positive to negative values. The temperature rise in the workpiece is very small. Overall temperature distribution in the chip is determined by plastic work 
dissipation in the chip rather than friction along the tool-chip interface [13].

\subsection{Plastic strain}

Equivalent plastic strain (PEEQ) lies in the range of 0.8 to 3 in most of the segmented section where intense localized deformation occurs; however, PEEQ reaches the value of 6 where elements are distorted or broken in the chip. The PEEQ results are consistent with the results obtained in [32] in which the simulations were performed for the machining of Ti6Al4V at $500 \mathrm{~m} /$ min.

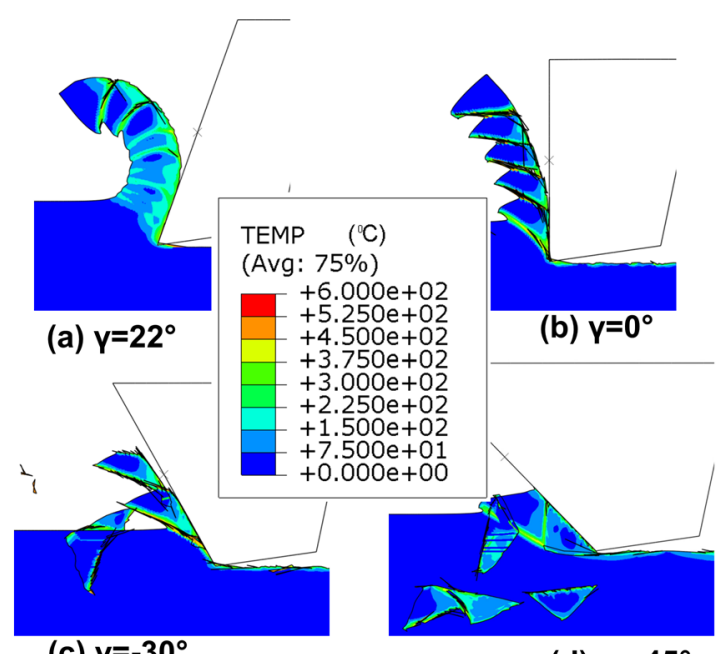

(c) $Y=-30^{\circ}$

(d) $Y=-45^{\circ}$

Fig. 9. Temperature changes during chip formation $\left(G_{f}=1500 \mathrm{~N} / \mathrm{m}, h=20 \mu \mathrm{m}\right)$

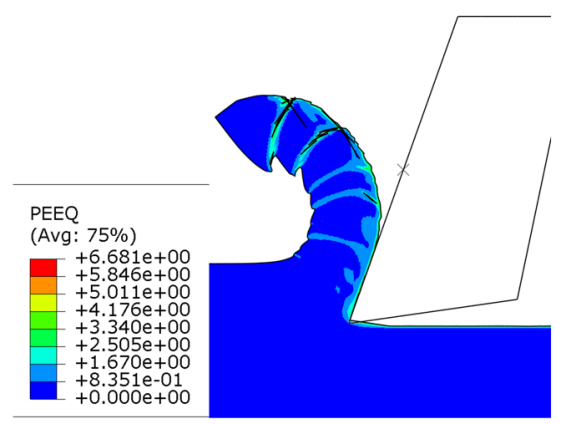

Fig. 10. Equivalent plastic strain demonstration $\left(\gamma=22^{\circ}, G_{f}=1500 \mathrm{~N} / \mathrm{m}, h=20 \mu \mathrm{m}\right)$

\section{CONCLUSIONS}

With the latest FEM models, a set of new results has been presented on the effects of influential material parameters and processing parameters on chip formation.
The morphology of cutting chips (continuous chip, discontinuous chip, and serrated chips) is simulated in Abaqus/Explicit finite element software. The Johnson-Cook constitutive material model is used in conjunction with the Johnson-Cook progressive damage model by using fracture energy as a damage evolution criterion. In addition, the fracture energybased damage evolution criterion is integrated into the model to reduce mesh dependency. According to simulation results, the fracture energy dissipation used for damage evolution is an influential factor in chip formation. As it was explained in preceding sections, the very lower values of fracture energy lead to discontinuous chip formation whereas the increasing values of fracture energy result in continuous chips with straighter chip shapes. Another essential factor in chip formation is the depth of cut. Though previous research did not demonstrate the significant effect of rake angle on the degree of chip segmentation, it has been found that a significant influence at smaller depths of cut. According to simulation results in this paper, with the increasing depth of cut chip type tends to become more segmented and finally serrated chips are generated. However, a great difficulty was encountered in the simulation with smaller depths of cut down to less a micron level due to the mesh size and computational power. In addition, the effects of rake angle in chip formation are simulated. Generated chips tend to be serrated and eventually the discontinuous type when the tool rake angle is moving from positive values to negative values. These findings are significant when a grinding case is to be studied, in which the depth of cut is smaller, and rake angles are much larger on the negative side.

In addition to the chip type and shape information obtained from the finite element simulation results, some mechanical parameters such as stress, strain, strain rate, temperature rise, which are very difficult to measure on the experimental test, can be obtained via finite element modelling of chip formation process.

Simulation results show that discontinuous chips are generated with very low fracture energy, while continuous chips are generated with increasing fracture energy. The chip shape is also changing from curling to straight with increasing fracture energy. High depth of cut results in serrated chip while the low depth of cut results in the continuous chip. Moreover, the transition from continuous to the discontinuous chip is observed when tool rake angle moves from positive values to negative values. 


\section{REFERENCES}

[1] Xie, J.Q., Bayoumi, A.E., Zbib, H.M. (1996). A study on shear banding in chip formation of orthogonal machining. International Journal of Machine Tools Manufacture, vol. 36, no. 7, p. 835-847, D0l:10.1016/0890-6955(95)00016-X.

[2] Mabrouki, T., Courbon, C., Zhang, Y., Rech, J., Nélias, D., J., Asad, M., Hamdi, H., Belhadi, S., Salvatore, F. (2016). Some insights on the modelling of chip formation and its morphology during metal cutting operations. Comptes Rendus Mécanique, vol. 344, no. 4-5, p. 335-354, D0l:10.1016/j. crme.2016.02.003.

[3] Batra, R.C., Liu, D.S. (1990). Adiabatic shear banding in dynamic plane strain compression of a viscoplastic material. International Journal of Plasticity, vol. 6, no. 2, p. 231-246, DOI:10.1016/0749-6419(90)90023-8.

[4] Sima, M., Özel, T. (2010). Modified material constitutive models for serrated chip formation simulations and experimental validation in machining of titanium alloy Ti-6Al-4V. International Journal of Machine Tools Manufacture, vol. 50, no. 11, p. 943-960, Dol:10.1016/j.ijmachtools.2010.08.004.

[5] Aurich, J.C., Bil, H. (2006). 3D finite element modelling of segmented chip formation. CIRP Annals - Manufacturing Technology, vol. 55, no. 1, p. 47-50, D0l:10.1016/\$00078506(07)60363-1.

[6] Calamaz, M., Coupard, D., Nouari, M., Girot F. (2011). Numerical analysis of chip formation and shear localisation processes in machining the Ti-6Al-4V titanium alloy. International Journal of Advanced Manufacturing Technology, vol. 52, no. 9, p. 887-895, D0l:10.1007/s00170-010-2789-x.

[7] Bäker, M., Rösler, J., Siemers, C. (2002). A finite element model of high speed metal cutting with adiabatic shearing. Computers and Structures, vol. 80, no. 5-6, p. 495-513, DOl:10.1016/S0045-7949(02)00023-8.

[8] Wang, B., Liu, Z. (2015). Shear localization sensitivity analysis for Johnson-Cook constitutive parameters on serrated chips in high speed machining of Ti6Al4V. Simulation Modelling Practice and Theory, vol. 55, p. 63-76, D0l:10.1016/j. simpat.2015.03.011.

[9] Strenkowski, J.S., Carroll, J.T. (1985). A finite element model of orthogonal metal cutting. Journal of Engineering for Industry, vol. 107, no. 4, p. 347-354, D0l:10.1115/1.3186008.

[10] Mamalis, A.G., Horváth, M., Branis, A.S., Manolakos, D.E. (2001). Finite element simulation of chip formation in orthogonal metal cutting. Journal of Materials Processing Technology, vol. 110, p. 19-27, D0l:10.1016/S09240136(00)00861-X.

[11] Huang, J.M., Black, J.T. (1996). An Evaluation of chip separation criteria for the FEM simulation of machining. Journal of Manufacturing Science and Engineering, vol. 188, no. 4, p. 545-554, D0I:10.1115/1.2831066.

[12] Bil, H., Kılıç, S.E., Tekkaya, A.E. (2004). A comparison of orthogonal cutting data from experiments with three different finite element models. International Journal of Machine Tools and Manufacture, vol. 44, no. 9, p. 933-944, D0l:10.1016/j. ijmachtools.2004.01.016.

[13] Shet, C., Deng, X. (2000). Finite element analysis of the orthogonal metal cutting process. Journal of Materials
Processing Technology, vol. 105, no. 1-2, p. 95-109, DOl:10.1016/S0924-0136(00)00595-1.

[14] Movahhedy, M.R., Gadala, M.S., Altintas, Y. (2000). Simulation of chip formation in orthogonal metal cutting process: An ALE finite element approach. Machining Science and Technology, vol. 4, no. 1, p. 15-42, Dol:10.1080/10940340008945698.

[15] Özel, T., Zeren, E. (2007). Finite element modelling the influence of edge roundness on the stress and temperature fields induced by high speed machining. International Journal of Advanced Manufacturing Technology, vol. 35, no. 3, p. 255267, D0l:10.1007/s00170-006-0720-2.

[16] ABAQUS/CAE User's Manual, Version 6.8, (2009). Simulia, Providence.

[17] Ambati, R., Yuan, H. (2010). FEM mesh-dependence in cutting process simulation. International Journal of Advanced Manufacturing Technology, vol. 53, no. 1, p. 313-323, DOl:10.1007/s00170-010-2818-9.

[18] Mabrouki, T., Girardin, F., Asad, M., Rigal, J.F. (2008). Numerical and experimental study of dry cutting for an aeronautic aluminium alloy (A2024-T351). International Journal of Machine Tools and Manufacture, vol. 48, no. 11, p. 1187-1197, D0l:10.1016/j.jimachtools.2008.03.013.

[19] ljaz, H., Zain-ul-abdein, M., Saleem, W., Asad, M., Mabrouki, T. (2016). A numerical approach on parametric sensitivity analysis for an aeronautic aluminium alloy turning process. Mechanika, vol. 22, no. 2, p. 149-155, D0l:10.5755/j01. mech.22.2.12825.

[20] Chen, G., Li, J., He, Y., Ren, C. (2014). A new approach to the determination of plastic flow stress and failure initiation strain for aluminum alloys cutting process. Computational Materials Science, vol. 95, p. 568-578, D0l:10.1016/j. commatsci.2014.08.029.

[21] Zhang, Y.C., Mabrouki, T., Nelias, D., Gong, Y.D. (2011). Chip formation in orthogonal cutting considering interface limiting shear stress and damage evolution based on fracture energy approach. Finite Elements in Analysis and Design, vol. 47, no. 7, p. 850-863, D0l:10.1016/j.finel.2011.02.016.

[22] Johnson, G.R., Cook, W.H. (1983). A constitutive model and data for metals subjected to large strains, high strain rates and high temperatures. Proceeding of the $7^{\text {th }}$ International Symposium on Ballistics, Hague, p. 541-547.

[23] Johnson, G.R., Cook, W.H. (1985). Fracture characteristics of three metals subjected to the various strains, strain rates, temperatures and pressures. Engineering Fracture Mechanics, vol. 21, no. 1, p. 31-48, D0l:10.1016/00137944(85)90052-9.

[24] Teng, X., Wierzbicki, T. (2006). Evaluation of six fracture models in high velocity perforation. Engineering Fracture Mechanics, vol. 73, no. 12, p. 1653-1678, Dol:10.1016/j. engfracmech.2006.01.009.

[25] Öpöz, T. T., Chen, X. (2012). Influential parameters in determination of chip shape in high speed machining. Key Engineering Materials, vol. 496, p. 211-216, D0l:10.4028/ www.scientific.net/KEM.496.211.

[26] Hillerborg, A., Modéer, M., Peterson, P.E. (1976). Analysis of crack formation and crack growth in concrete by means of fracture mechanics and finite elements. Cement and Concrete 
Research, vol. 6, no. 6, p. 773-781, D0l:10.1016/00088846(76)90007-7.

[27] He, M., Li, F. (2010). Modified transformation formulae between fracture toughness and CTOD of ductile metals considering pre-deformation effects. Engineering Fracture Mechanics, vol. 77, no. 14, p. 2763-2771, D0l:10.1016/j. engfracmech.2010.06.021.

[28] Garrison, Jr W.M., Moody, N.R. (1987). Ductile fracture. Journal of Physics and Chemistry of Solids, vol. 48, no. 11, p. 1035-1047, D0l:10.1016/0022-3697(87)90118-1.

[29] Sisto, T.S.D., Carr, F.L., Larson, F.R. (1964). The influence of section size on the mechanical properties and fracture toughness of 7075-T6 aluminum, 6Al-6V-2Sn titanium, and AISI 4340 steel. Material testing laboratory U.S. Army Materials Research Agency Watertown, Massachusetts.
[30] Belhadi, S., Mabrouki, T., Rigal, J-F., Boulanouar, L. (2005). Experimental and numerical study of chip formation during straight turning of hardened AISI 4340 steel. Proceedings of the Institution of Mechanical Engineers, Part B: Journal of Engineering Manufacture, vol. 219, no. 7, p. 515-524, DOl:10.1243/095440505X32445.

[31] Haddag, B., Atlati, S., Nouari, M., Barlier, C., Zenasni, M. (2012). Analysis of the Cutting Parameters Influence During Machining Aluminium Alloy A2024-T351 with Uncoated Carbide Inserts. Engineering Transactions, vol. 60, no. 1, p. 31-39.

[32] Wang, B., Liu, Z. (2015). Shear localization sensitivity analysis for Johnson-Cook constitutive parameters on serrated chips in high speed machining of Ti6Al4V. Simulation Modelling Practice and Theory, vol. 55, p. 63-76, D0l:10.1016/j. simpat.2015.03.011. 